\title{
Quantified Complementary and Alternative Medicine: Convergence of Digital Health Technologies and Complementary and Alternative Medicine
}

\author{
Nico Steckhan ${ }^{a, b}$ Bert Arnrich ${ }^{b}$ \\ anstitute for Social Medicine, Epidemiology and Health Economics, Charité - Universitätsmedizin Berlin, \\ Freie Universität Berlin, Humboldt-Universität zu Berlin, and Berlin Institute of Health, Berlin, Germany; \\ ${ }^{b}$ Digital Health Center, Hasso Plattner Institute, University of Potsdam, Potsdam, Germany
}

In recent years, consumer health technologies including wearables, Internet-of-Things, and health apps that support wellness and healthy lifestyle have emerged rapidly [1]. However, only a few of these new technologies have been certificated as medical product yet (e.g., only 40 CE-certified apps in Germany as of July 2019).

Common application scenarios include lifestyle-associated aspects like diet, exercise, stress management, or combinations of it. Interestingly, the same aspects are addressed by several fields of complementary and alternative medicine (CAM) like integrative or Mind Body Medicine. This synergy has mainly two reasons, first the modern technologies are getting better to process lifestyle data, and second, the regulations (Medical Device Regulation) are more complex if one includes non-wellness health claims (e.g., dosing of medications, medical decisions).

In 2020, the German Medical Device Directive will increase the risk classification for apps and health services implying any medical purpose, thus many lifestyle technologies need a certification comparable to other pharmacological or medical products (Federal Institute for Drugs and Medical Devices). Hence, technologies that are "just software" should be clinically investigated comparable to invasive medical products. This yields several opportunities and challenges for a patient-generated datadriven CAM.

Still medical sampling has a snapshot approach lacking the dynamical behaviour of a person's physiology.
Sensor technologies are able to provide metrics by means of active (prompted) or passive (unnoticed) measurements, offering considerable flexibility in approach. Those high-frequency longitudinal data sets can then be used for characterisation of a disease [2]. This yields a solution to the often acclaimed subjectivity of patient-reported outcome, that is common not only in CAM research. Further, mood states could be assessed using different sensor technologies. Even if this may be an indirect measure of one's psychological state, we believe we now have better tools at hand to quantify, for example, stress in real time $[3,4]$.

In order to apply such technologies in medicine, however, methods are needed that are also suitable for research beyond big players and big data (e.g., Apple, Google, and Samsung), such as in complementary medical research with small numbers of cases. The support from validated models and tools can be a beneficial ingredient if not transforming complementary medicine research (think of the one channel ECG in Apple Watch). For example, monitoring vital signs in real time and in daily life can dramatically enhance the field of research [5].

Thus, passive data collection using sensors in wristbands has several advantages: mainly, the high frequency or even continuous data acquisition, and further, better objectivity (not influenced by patient reporting and learning effects) can be achieved. 
As doctors spend more time with administrational work rather than talking with their patients, we have an information bias that could be circumvented if we had the right tools to effectively summarise a patient's health status. Providing longitudinal disease-related digital biomarkers to a healthcare practitioner to allow for objective and continuous clinical evaluation of a person's lifestyle could be a necessary step towards a more holistic medical understanding not only in CAM [2].

Journals like Nature - Digital Medicine or Journal of Medical Internet Research have become popular in this field. Recently, also The Lancet started a digital health sub journal. Published papers show that not only health services have to be established but rather research on socalled digital biomarkers is needed. Finally, establishing the basic research of digital health.

Also these digital biomarkers are more in line with the phenomenological understanding of behavioural therapies. The quantification of behavioural and consciousness involving processes can assist squaring the circle of qualia-related outcome $[6,7]$.

Monitoring and modelling biomedical, healthcare, and wellness data from individuals and converging data on a population scale have tremendous potential to improve understanding of chronic non-communicable diseases [8]. This will also improve primary care and will help to quantify transitions from healthy to diseased state, thus strengthening the importance of prevention strategies already before classical biomarkers hit thresholds [9].

If we have such monitoring systems, we are allowed to analyse such data in different contexts, thus enabling later options for early diagnosis and treatment.

Forecasting of health relevant events by using artificial intelligence has the ability to intervene early with lifestyle changes or more gentle medical approaches [10].

Whether the claimed improvements based on predictions such as better prevention and therapy adherence rates will help reduce hospital admission and readmission levels and doctor's visits remains to be proven.

Inevitably, these technologies have limitations that need to be dealt with. Some people will argue that selfawareness will not be achieved using external devices and more technical feedback loops/systems. On the other hand, it could be contradictory to distress patients with technical problems (e.g., battery life, connectivity), for example, during stress assessments. Depart from this, data privacy will become a major risk concerning the probably most sensitive data of a person.

Cooperation between start-ups and health insurance companies is growing, with the legal framework taking shape and a number of health apps already in Germany's reimbursement scheme. Most of the available mobile health apps focus on relaxation, mindfulness, and meditation. Whether an app like Headspace or 7Mind can achieve the same effect as a mind body therapy or how it can support Mindfulness-Based Stress Reduction has yet to be evaluated. The ability to intervene and measure at the same time, making interactions in real time accessible is of great value. One may think of the already established continuous glucose monitoring systems that help to not only improve quality of life but further excels in optimising glucose control of diabetic patients.

Longitudinal quantification of the individual-level human phenotype in situ using data from personal digital devices has established the so-called digital phenotyping.

In general, an efficient digital phenotyping can be a potential resource for a more lifestyle-concerned medicine. Especially when one realizes that Western diseases, such as coronary heart diseases, are associated with over $80 \%$ participation of lifestyle. These chronic non-communicable diseases will create a massive burden for health systems, including insurances, in the coming years.

Having quantifiable measures of one's lifestyle could transform healthcare into the direction of a preventive and lifestyle concerned medicine. What especially is of importance if we address non-communicable diseases and like to monitor adherence. New methods have to be developed to approach disease forecasting, detection, and monitoring. Those data will then enable a more quantifiable lifestyle-related picture as often suggested by several holistic approaches like traditional medical systems (e.g., Traditional European Medicine, Traditional Chinese Medicine, and Ayurveda). The rise of interdisciplinary research like systems medicine and digital health bridges the way for addressing the complexity and dynamics of multifactorial diseases, in such that CAM becomes more relevant for the scientific zeitgeist of these days.

We may not need to transform patients to quantified selves but introduce a whole new dimension to modern health research, prevention, and disease management.

\section{Acknowledgement}

We would like to acknowledge Jonas Chromik for reading the final draft.

\section{Disclosure Statement}

The authors have no conflicts of interest to declare.

\section{Funding Sources}

No funding sources received.

\section{Author Contributions}

N. Steckhan has written the manuscript and B. Arnrich edited it. 


\section{References}

1 Peake JM, Kerr G, Sullivan JP. A Critical Review of Consumer Wearables, Mobile Applications, and Equipment for Providing Biofeedback, Monitoring Stress, and Sleep in Physically Active Populations. Front Physiol. 2018 Jun;9:743

2 Kourtis LC, Regele OB, Wright JM, Jones GB. Digital biomarkers for Alzheimer's disease: the mobile/wearable devices opportunity [Internet]. Vol. 2, npj Digital Medicine. 2019. Available from: http://dx.doi.org/https://doi. org/10.1038/s41746-019-0084-2.

3 Goodday SM, Friend S. Unlocking stress and forecasting its consequences with digital technology. NPJ Digit Med. 2019 Jul;2(1):75.
4 Smets E, Rios Velazquez E, Schiavone G, Chakroun I, D'Hondt E, De Raedt W, et al. Large-scale wearable data reveal digital phenotypes for daily-life stress detection. NPJ Digit Med. 2018 Dec;1(1):67.

5 Shameer K, Badgeley MA, Miotto R, Glicksberg BS, Morgan JW, Dudley JT. Translational bioinformatics in the era of real-time biomedical, health care and wellness data streams. Brief Bioinform. 2017 Jan;18(1): $105-24$.

6 Jacobson NC, Weingarden H, Wilhelm S. Digital biomarkers of mood disorders and symptom change. NPJ Digit Med. 2019 Feb; 2(1):3.
7 Baumeister H, Montag C. Digital Phenotyping and Mobile Sensing: New Developments in Psychoinformatics. Springer Nature; 2019. https://doi.org/10.1007/978-3-030-31620-4.

8 Bhavnani SP, Narula J, Sengupta PP. Mobile technology and the digitization of healthcare. Eur Heart J. 2016 May;37(18):1428-38.

9 Price ND, Magis AT, Earls JC, Glusman G, Levy R, Lausted C, et al. A wellness study of 108 individuals using personal, dense, dynamic data clouds. Nat Biotechnol. 2017 Aug; 35(8):747-56

10 Norgeot B, Glicksberg BS, Trupin L, Lituiev D, Gianfrancesco M, Oskotsky B, et al. Assessment of a Deep Learning Model Based on Electronic Health Record Data to Forecast Clinical Outcomes in Patients With Rheumatoid Arthritis. JAMA Netw Open. 2019 Mar; 2(3):e190606 